\title{
A Policy Statement of the Society of General Internal Medicine on Tackling Racism in Medical Education: Reflections on the Past and a Call to Action for the Future
}

\author{
Eloho Ufomata, $M D, M S^{1}$ (D), Sarah Merriam, $M D, M S^{1,2}$, Aditi Puri, $M D, M S^{3}$, \\ Katherine Lupton, $M D^{4}$, Darlene LeFrancois, $M D^{5}$, Danielle Jones, $M D^{6}$, \\ Attila Nemeth, $\mathrm{MD}^{7}$, Laura K. Snydman, $\mathrm{MD}^{8}$, Rachel Stark, MD, $M P H^{9}$, and \\ Carla Spagnoletti, MD, $\mathrm{MS}^{\top}$
}

\begin{abstract}
'Division of General Internal Medicine, University of Pittsburgh School of Medicine, Pittsburgh, PA, USA; ${ }^{2}$ Division of General Internal Medicine, VA Pittsburgh Healthcare System, Pittsburgh, PA, USA; ${ }^{3}$ Division of Hospital Medicine, Northwestern Feinberg School of Medicine, Chicago, IL, USA; ${ }^{4}$ Division of General Internal Medicine at Zuckerberg San Francisco General, University of California San Francisco, San Francisco, CA, USA; ${ }^{5}$ Division of General Internal Medicine, Montefiore Medical Center, Albert Einstein College of Medicine, Bronx, NY, USA; ${ }^{6}$ Division of General Medicine and Geriatrics, Emory University School of Medicine, Atlanta, GA, USA; ${ }^{7}$ Division of Acute Medicine, VA Northeast Ohio Healthcare System, Cleveland, Ohio, USA; ${ }^{8}$ Division of General Internal Medicine, Tufts Medical Center, Boston, MA, USA; ${ }^{9}$ Department of Medicine, Cambridge Health Alliance, Harvard Medical School, Cambridge, MA, USA.
\end{abstract}

J Gen Intern Med 36(4):1077-81

DOI: $10.1007 / \mathrm{s} 11606-020-06445-2$

(c) Society of General Internal Medicine 2021

\section{INTRODUCTION}

Structural racism, or the practices, policies, and norms that perpetuate white supremacy, is ubiquitous within US social systems, including in institutions of medical education. ${ }^{1,2}$ The call for medical educators to design and implement targeted anti-racist curricula is not new, ${ }^{3-9}$ yet formal integration of anti-racism education aimed at dismantling systemic racism remains underemphasized in undergraduate and graduate medical education. ${ }^{10}$ Unconscious bias and cultural competency curricula are popular approaches to addressing issues of diversity and inclusion. However, these frameworks ${ }^{11,12}$ have been critiqued as oversimplifying culture, propagating stereotypes, providing a superficial understanding of the impact of race and identity, and overlooking structural inequities and issues of privilege. ${ }^{2,4,7,13,14}$ We must progress beyond these overly simplistic approaches to directly combat racism and structural oppression. Here, we focus a critical lens on the manifestations of structural racism embedded in institutions of medical education for the purpose of identifying the multidimensional strategies necessary to combat it.

\section{SETTING THE STAGE}

\section{The Historic Color Line}

Historically, Black Americans were barred from medical training in the USA. This "color line" forced Black Americans to

Received October 2, 2020

Accepted December 10, 2020

Published online January 22, 2021 seek education in Europe, including the first Black US physician, Dr. James McCune Smith-who graduated from the University of Glasgow in $1837 .{ }^{15}$ The Flexner report of 1910, hailed as a transformational catalyst in US medical education, also led to the closure of multiple Black medical colleges and further limited access to training for Black physicians. ${ }^{16,17}$ Just 60 years ago, the color line, or the systematic exclusion of Black Americans from most medical institutions in the USA, still held strong (Fig. 1). ${ }^{18}$

\section{The Current Color Line}

Students from racial and ethnic groups traditionally underrepresented in medicine (URiM), particularly Black, LatinX, and Indigenous people, encounter substantial structural racism from an early age. They are more likely to live in low-income communities than their white counterparts, ${ }^{19,20}$ and subsequently attend underfunded schools with high teacher turnover and fewer advanced placement courses, ${ }^{21,22}$ which leads to racial disparities in standardized exam scores and attainment of higher education. ${ }^{19,23}$ URiM students confront an "amplification cascade" 24 of disadvantage: colleges, medical schools, and residency programs place disproportionate value on traditional academic metrics, limiting URiM students' progress at every step along the medical education pipeline.

\section{Racism in Medical Education Today}

Structural racism negatively affects the educational experiences of URiM students, and permeates the formal, informal, and hidden curricula of medical education.

URiM students continue to face institutionalized roadblocks that negatively impact their ability to succeed during their medical training. There is a dearth of Black faculty in US medical schools compared to the general population: $3.6 \%$ of medical school faculty identify as Black, compared with $13 \%$ 


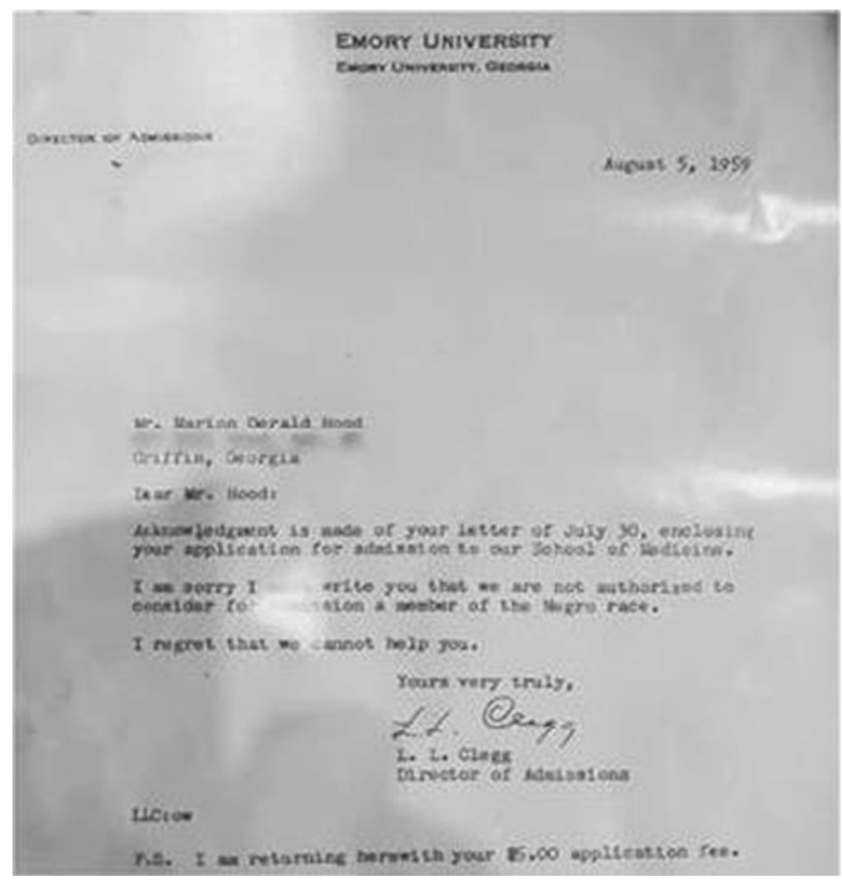

Fig. 1 The historic color line. Rejection letter from the Director of Admissions, Emory University, to Marion Gerald Hood, Aug. 5, $1959 .^{18}$

of Americans who identified as Black or African American in $2018 .^{25}$ Without adequate exposure to faculty like themselves, URiM students may not receive the role-modeling needed to cultivate their aspirations. ${ }^{26,27}$ Similarly, overemphasis on clinical grading and honors, which has been shown to be discriminatory, impacts URiM students' ability to be competitive in their residency applications. ${ }^{24,28-30}$ On the Medical Student Performance Evaluation (MSPE), Black medical students are more frequently described as "competent," which is less likely to have positive connotation for Black students, in comparison with their white counterparts who are more likely to be described as "outstanding" or "exceptional.",30

As educators, we are guilty of furthering racist messages by perpetuating scientific inaccuracies. We teach trainees to apply "evidence-based" medicine to patients of color despite the fact that most evidence is incomplete and fails to include them. ${ }^{31}$ This discounts the importance of including patients of color in research and may affect the quality of the medical care we provide. Similarly, we reinforce racist ideology when we ignore the truth that intra-racial genetic differences are greater than interracial differences, ${ }^{32}$ and attribute a biological basis to racial and ethnic differences in disease rates and outcomes. As a result, students may not understand that race is a social construct and that these differences are due to the disproportionate impact of structural racism leading to worse social determinants of health $(\mathrm{SDOH}){ }^{1,33}$

We also exhibit bias that affects clinical care and education. For example, while precepting resident patient encounters, we are more likely to call Black patients "non-compliant" or assume that they "don't care" about their health when discussing reasons for lower adherence to medical recommendations, rather than exploring the SDOH caused by structural racism. ${ }^{34}$ Other examples of this hidden curriculum show up in the classroom: we often assume a default (white) race by omitting race identifiers for white patients, associate other racial identifiers with specific diseases, and perpetuate knowledge gaps and stigma by showing medication-related rashes on patients with white skin while showing manifestations of syphilis on patients with black skin for example. ${ }^{6,35}$

From an organizational perspective, it is important to note that despite increasing calls for dedicated curricular emphasis on structural racism, social justice, and $\mathrm{SDOH},{ }^{36}$ no clear standards exist within either undergraduate medical education (UME) or graduate medical education (GME) to guide these efforts. Although the Liaison Committee on Medical Education (LCME) accreditation requirements state that medical school curricula must include teaching on "societal problems, cultural competence, and healthcare disparities," $" 37$ there is no mention of structural racism or the impact of racism on patients and communities.

\section{Existing Anti-racist Curricula}

Most published anti-racist curricula are elective and offered at the UME level, ${ }^{38-42}$ with fewer curricula geared towards GME learners, ${ }^{40,41}$ faculty, ${ }^{40,43,44}$ or audiences comprised of a spectrum of rank and expertise. ${ }^{43,45}$ Despite recognition that is robust, dedicated faculty training is a prerequisite for effective curricular development and implementation, ${ }^{4}$ faculty development efforts focused on anti-racist teaching, and dialoguing about race, racism, and white privilege are scarce. Some important work has been done with respect to interpersonal manifestations of racism, like supporting trainees in the face of microaggressions and mistreatment ${ }^{45,46}$ and acknowledging implicit bias. $^{47}$

Indeed, though curricula that focus on individual-level factors (implicit bias and microaggressions) may increase awareness of the negative experiences of URiM trainees and physicians, this "consciousness raising" 48 can create a false perception of change if the increased dialogue about interpersonal bias is not accompanied by efforts to identify and correct the systemic racist structures present within our institutions. In addition, URiM faculty and trainees are often asked or expected to conduct these trainings without adequate compensation. This undervaluing leads to a minority tax of additional tasks beyond the duties expected of their roles. ${ }^{49}$

\section{A CALL TO ORGANIZATIONAL ACTION}

We recommend a multidimensional organizational strategy to further the goal of combating structural racism and achieving social justice and racial equity in our institutions of medical education. 


\section{Professional Culture}

A culture of inclusion reinforces that URiM physicians and trainees are viewed as outsiders. Rather than generously "including" all, we must change a system that is exclusionary and actively adopt the following anti-racist strategies:

- Reflect on the resilience of our trainees and faculty of color and gain an understanding of the profound depth of structural disadvantage that impacts communities of color, and subsequently, learners from URiM backgrounds.

- Acknowledge that the institutions of medicine and medical education are grounded in white, upper-middleclass norms and challenge them by adopting a lens of cultural humility.

- Highlight the contributions of URiM individuals to scientific discovery and the practice of medicine and display URiM individuals and decrease visibility of individuals with racist affiliations at our institutions of learning. ${ }^{50,51}$

- Normalize diverse cultures within medicine and medical education and ensure that our definitions of professionalism incorporate cultural diversity in modes of language and communication, dress, hairstyles, and other forms of expression, rather than expecting assimilation to Eurocentric norms.

- Act as advocates instead of bystanders. White faculty should call out microaggressions and discrimination in the educational environment. ${ }^{26,27,52}$

\section{Learner Support and Academic Environment}

To create a more supportive environment for URiM individuals at all levels in medicine, and to reduce alienation and leaks in the pipeline, we must:

- Mitigate the impacts of stereotype threat by creating an identity-safe environment which does not trigger performance anxiety in URiM learners, but rather is encouraging and focuses on a growth mindset. ${ }^{53-55}$

- Counteract our own biases towards URiM learners by considering counter-stereotypical exemplars ${ }^{56}$ and utilizing strong descriptors in evaluations and supportive documentation for applications.

- Ensure that the bodies that control entry to medical training and practice, including admissions, promotion, and hiring committees, have adequate representation of URiM individuals, while simultaneously avoiding an increase in minority tax by compensating trainees and faculty adequately for these activities.

- Place academic value on anti-racism efforts through methods that are not traditionally viewed as scholarship for the purposes of admission, promotion, and leadership for all trainees and faculty.
- Ensure strategic mentorship, sponsorship, and coaching of URIM trainees and physicians to dismantle the color lines that block access into medical education and subsequent ascension into leadership positions.

\section{Self-appraisal and Educational Policy}

Several recent publications outline essential steps that individuals and institutions should take to better address racism and strive for racial justice in medicine. ${ }^{50,57}$ Drawing from this literature, we recommend that the education leaders at each institution:

- Conduct an internal review of how race and racialized disparities are presented within existing curricula. ${ }^{58}$

- Apply an evidence-based tool to examine the portrayal of race and structural inequities within clinical teaching cases. ${ }^{59}$

- Conduct intensive faculty development about race and racism. ${ }^{60}$

- Train educators how to teach, assess, and develop curricula using an anti-racist equity lens. ${ }^{4,60}$

- Incorporate a framework to promote equity and inclusion for URiM students. ${ }^{53}$

- Adopt a holistic model to generate equity in awards selection. ${ }^{61}$

- Report on pre-defined metrics of success in a transparent and accessible manner.

\section{Furthering Anti-racist Curricula}

First, we define anti-racist curricula in medical education as pedagogy that includes explicit instruction on the history and continuation of racism in medicine, and is transformational in creating a paradigm shift to combat further perpetuation of racist ideologies. ${ }^{62}$ To support that transformation, we promote:

- The importance of the redefining race as a social construct in medical education curricula as highlighted in a recent student perspective. ${ }^{1}$

- The utilization of critical race theory as a guiding framework to educate learners about the etiology of healthcare disparities. ${ }^{2}$

- A longitudinal instructional model with the goal of mitigating the impact of structural racism at both UME and GME levels.

- A new core competency in medical education "mastering the health effects of structural racism," ${ }^{, 63}$ and encourage the LCME and Accreditation Council for Graduate Medical Education (ACGME) to create of actionable objectives and competencies towards this goal, which would standardize a minimum expectation of learner competency.

- This will catalyze medical schools and training programs to develop measurable learning objectives on structural 
racism, the curricular materials to teach about it, and the evaluation and assessment structures to ensure that learners attain this knowledge.

- "A Toolkit for Teaching About Racism in the Context of Persistent Health and Healthcare Disparities" as a starting point. These concrete steps can form the foundation for curriculum design about identity, intersectionality, and privilege.

\section{CONCLUSION}

Medical educators are entrusted to train a workforce of doctors to care for our diverse population. To date, we have failed to meet our public responsibility to identify, address, and actively combat structural racism in medical education. We must do better. The road ahead is not easy nor is the endpoint shortterm. In order to demolish structural racism in medical education, we must adopt an actively anti-racist framework. Most importantly, we must shift the paradigm from the goal of fostering diversity to a goal of dismantling a system that fosters exclusion and rebuild a system that enables all the individuals within it to thrive.

We are optimistic a multidimensional strategy that promotes deep institutional reflection on professional and academic culture incorporates an anti-racist approach to the formal, informal, and hidden curricula; provides intensive faculty development; and reverses discriminatory organizational policies will provide an opportunity for the institutions of medical education to change course and adopt the values of social justice and racial equity. It is our strong hope that all members of the wider medical education community will join us in this call to action.

Acknowledgments: This perspective, authored by several members of the Society of General Internal Medicine's Education Committee, was endorsed by the Education Committee, The Health Equity Commission, and the Executive Committee of the Society of General Internal Medicine. We would also like to thank Ethan Lennox, MA, Division of General Internal Medicine, University of Pittsburgh, for his contributions.

Corresponding Author: Eloho Ufomata, MD, MS; Division of General Internal Medicine, University of Pittsburgh School of Medicine, Pittsburgh, PA, USA (e-mail: ufomataeo@upmc.edu).

\section{REFERENCES}

1. Nieblas-Bedolla E, Christophers B, Nkinsi NT, Schumann PD, Stein E. Changing How Race Is Portrayed in Medical Education: Recommendations From Medical Students. Academic Medicine. 9000;Publish Ahead of Print. https://doi.org/10.1097/acm.0000000000003496

2. Tsai J, Crawford-Roberts A. A Call for Critical Race Theory in Medical Education. Academic Medicine. 2017;92(8):1072-3. https://doi.org/10. 1097/acm.0000000000001810

3. Ahmad NJ, Shi M. The Need for Anti-Racism Training in Medical School Curricula. Academic medicine : journal of the Association of American Medical Colleges. 2017;92(8):1073. https://doi.org/10.1097/acm. 0000000000001806
4. Acosta D, Ackerman-Barger $\mathbf{K}$. Breaking the Silence: Time to Talk About Race and Racism. Academic medicine : journal of the Association of American Medical Colleges. 2017;92(3):285-8. https://doi.org/10.1097/ acm.0000000000001416

5. Wear D, Zarconi J, Aultman JM, Chyatte MR, Kumagai AK. Remembering Freddie Gray: Medical Education for Social Justice. Academic medicine : journal of the Association of American Medical Colleges. 2017;92(3):312-7. https://doi.org/10.1097/acm.0000000000001355

6. Brooks KC. A piece of my mind. A silent curriculum. Jama. 2015;313(19):1909-10. https://doi.org/10.1001/jama.2015.1676

7. Kumagai AK, Lypson ML. Beyond cultural competence: critical consciousness, social justice, and multicultural education. Academic medicine : journal of the Association of American Medical Colleges. 2009;84(6):782-7. https://doi.org/10.1097/ACM.0b013e3181a42398

8. Tervalon M, Murray-Garcia J. Cultural humility versus cultural competence: a critical distinction in defining physician training outcomes in multicultural education. Journal of health care for the poor and underserved. 1998;9(2):117-25. https://doi.org/10.1353/hpu.2010. 0233

9. Bassett MT. \#BlackLivesMatter - A Challenge to the Medical and Public Health Communities. New England Journal of Medicine. 2015;372(12): 1085-7. https://doi.org/10.1056/NEJMp1500529

10. White SO, O. Coverage of Racial Disparity in Medicine Education Content in Accredited U.S. Medical Schools, Academic Year 2017-2018. AAMC Curriculum Inventory Report. 2019.

11. Karani R, Varpio L, May W, Horsley T, Chenault J, Miller KH, et al. Commentary: Racism and Bias in Health Professions Education: How Educators, Faculty Developers, and Researchers Can Make a Difference. Academic medicine : journal of the Association of American Medical Colleges. 2017;92(11S Association of American Medical Colleges Learn Serve Lead: Proceedings of the 56th Annual Research in Medical Education Sessions):S1-s6. https://doi.org/10.1097/acm. 0000000000001928

12. Gliatto P, Karani R. Viewpoint From 2 Undergraduate Medical Education Deans The Residency Application Process: Working Well, Needs Fixing, or Broken Beyond Repair? Journal of Graduate Medical Education. 2016;8(3):307-10. https://doi.org/10.4300/JGME-D-16-00230.1

13. Wear D. Insurgent multiculturalism: rethinking how and why we teach culture in medical education. Academic medicine : journal of the Association of American Medical Colleges. 2003;78(6):549-54. https:// doi.org/10.1097/00001888-200306000-00002

14. Brooks KC, Rougas S, George $\mathbf{P}$. When Race Matters on the Wards: Talking About Racial Health Disparities and Racism in the Clinical Setting. MedEdPORTAL. 2016;12. https://doi.org/10.15766/mep_ 2374-8265. 10523

15. Harley EH. The forgotten history of defunct black medical schools in the 19th and 20th centuries and the impact of the Flexner Report. J Natl Med Assoc. 2006;98(9):1425-9.

16. Flexner A. Medical education in the United States and Canada. From the Carnegie Foundation for the Advancement of Teaching, Bulletin Number Four, 1910. Bull World Health Organ. 2002;80(7):594-602.

17. Duffy TP. The Flexner Report-100 years later. Yale J Biol Med. 2011;84(3):269-76.

18. Clegg L. L. (Director of Admissions, Emory University). Letter to: Marion Gerald Hood. 1959, Aug 5. Located at: https://catscontentwriting.com/ dr-hoods-rejection-letter/

19. Lucey CR, Saguil A. The Consequences of Structural Racism on MCAT Scores and Medical School Admissions: The Past Is Prologue. Academic Medicine. 2020;95(3):351-6. https://doi.org/10.1097/acm. 0000000000002939

20. National Center for Children in Poverty. United States Demographics of Low-Income Children. 2018.

21. Darling-Hammond L. Unequal Opportunity: Race and Education. Brookings Institute Report.

22. Education and Socioeconomic Status. American Psychological Association.

23. Gates MA. Civil Rights Challenge to Standardized Testing in College Admissions. Amicus: Harvard Civil Rights - Civil Liberties Law Review. 2019.

24. Teherani A, Hauer KE, Fernandez A, King TE, Jr., Lucey C. How Small Differences in Assessed Clinical Performance Amplify to Large Differences in Grades and Awards: A Cascade With Serious Consequences for Students Underrepresented in Medicine. Academic medicine : journal of the Association of American Medical Colleges. 2018;93(9):1286-92. https://doi.org/10.1097/acm.0000000000002323 
25. Diversity in medicine: facts and figures 2019. Association of American Medical Colleges. 2019.

26. Bullock JL, Lockspeiser T, del Pino-Jones A, Richards R, Teherani A, Hauer KE. They Don't See a Lot of People My Color: A Mixed Methods Study of Racial/Ethnic Stereotype Threat Among Medical Students on Core Clerkships. Academic Medicine. 2020;Publish Ahead of Print

27. Ackerman-Barger $\mathbf{K}$, Boatright $\mathbf{D}$, Gonzalez-Colaso $\mathbf{R}$, Orozco $\mathbf{R}$, Latimore D. Seeking Inclusion Excellence: Understanding Racial Microaggressions as Experienced by Underrepresented Medical and Nursing Students. Academic medicine : journal of the Association of American Medical Colleges. 2020;95(5):758-63. https://doi.org/10 1097/acm.0000000000003077

28. Boatright D, Ross D, O'Connor P, Moore E, Nunez-Smith M. Racial Disparities in Medical Student Membership in the Alpha Omega Alpha Honor Society. JAMA internal medicine. 2017;177(5):659-65. https:// doi.org/10.1001/jamainternmed.2016.9623

29. Low D, Pollack SW, Liao ZC, Maestas R, Kirven LE, Eacker AM, et al. Racial/Ethnic Disparities in Clinical Grading in Medical School. Teaching and learning in medicine. 2019;31(5):487-96. https://doi.org/10.1080/ 10401334.2019.1597724

30. Ross DA, Boatright D, Nunez-Smith M, Jordan A, Chekroud A, Moore EZ. Differences in words used to describe racial and gender groups in Medical Student Performance Evaluations. PLoS ONE. 2017;12(8):e0181659. https://doi.org/10.1371/journal.pone.0181659

31. Terry M. Pushing for More Ethnic and Racial Diversity in Clinical Trials. Biospace. 2020.

32. Rosenberg NA. A population-genetic perspective on the similarities and differences among worldwide human populations. Human biology. 2011;83(6):659-84. https://doi.org/10.3378/027.083.0601

33. Worrall s. Why Race Is Not a Thing, According to Genetics. National Geographic. 2017.

34. Hall WJ, Chapman MV, Lee KM, Merino YM, Thomas TW, Payne BK, et al. Implicit Racial/Ethnic Bias Among Health Care Professionals and Its Influence on Health Care Outcomes: A Systematic Review. American journal of public health. 2015;105(12):e60-e76. https://doi.org/10. 2105/AJPH.2015.302903

35. Nolen L. How Medical Education Is Missing the Bull's-eye. New England Journal of Medicine. 2020;382(26):2489-91. https://doi.org/10.1056/ NEJMp 1915891

36. Siegel J, Coleman DL, James T. Integrating Social Determinants of Health Into Graduate Medical Education: A Call for Action. Academic Medicine. 2018;93(2):159-62. https://doi.org/10.1097/acm. 0000000000002054

37. LCME Functions and Structure of a Medical School - Standards for Accreditation of Medical Education Programs Leading to the MD Degree. 2020.

38. DallaPiazza M, Padilla-Register M, Dwarakanath M, Obamedo E, Hill J, Soto-Greene M. Exploring Racism and Health: An Intensive Interactive Session for Medical Students. MedEdPORTAL. 2018;14. https://doi.org/ 10.15766/mep_2374-8265.10783

39. Bereknyei S, Foran SM, Johnson KD, Scott AJ, Miller TL, Braddock CH. Stopping Discrimination Before It Starts: The Impact of Civil Rights Laws on Healthcare Disparities - A Medical School Curriculum. MedEdPORTAL. 2009;5. 10.15766/mep_2374-8265.7740

40. Chow CJ, Case GA, Matias CE. Tools for Discussing Identity and Privilege Among Medical Students, Trainees, and Faculty. MedEdPORTAL. 2019;15:10864. 10.15766/mep_2374-8265.10864

41. Medlock M, Weissman A, Wong SS, Carlo A, Zeng M, Borba C, et al. Racism as a Unique Social Determinant of Mental Health: Development of a Didactic Curriculum for Psychiatry Residents. MedEdPORTAL : the journal of teaching and learning resources. 2017;13:10618. https://doi. org/10.15766/mep_2374-8265.10618

42. Barkley L, Alford D. Medical Ethics and Health Equity: The Henrietta Lacks Story. MedEdPORTAL. 2015;11. https://doi.org/10.15766/mep_ 2374-8265. 10276

43. Neff J, Holmes SM, Knight KR, Strong S, Thompson-Lastad A McGuinness C, et al. Structural Competency: Curriculum for Medical Students, Residents, and Interprofessional Teams on the Structural Factors That Produce Health Disparities. MedEdPORTAL. 2020;16. https://doi.org/10.15766/mep_2374-8265.10888

44. Nelson SC, Prasad S, Hackman HW. Training providers on issues of race and racism improve health care equity. Pediatric blood \& cancer. 2015;62(5):915-7. https://doi.org/10.1002/pbc.25448
45. Sotto-Santiago S, Mac J, Duncan F, Smith J. "I Didn't Know What to Say": Responding to Racism, Discrimination, and Microaggressions With the OWTFD Approach. MedEdPORTAL : the journal of teaching and learning resources. 2020;16:10971-. https://doi.org/10.15766/mep_ 2374-8265.10971

46. Wilkins KM, Goldenberg MN, Cyrus KD. ERASE-ing Patient Mistreatment of Trainees: Faculty Workshop. MedEdPORTAL : the journal of teaching and learning resources. 2019;15:10865-. https://doi.org/10. 15766/mep_2374-8265.10865

47. Capers QI. How Clinicians and Educators Can Mitigate Implicit Bias in Patient Care and Candidate Selection in Medical Education. ATS Scholar.0(0):ats-scholar.2020-0024PS. https://doi.org/10.34197/atsscholar.2020-0024PS

48. Grundy S. The False Promise of Anti-racism Books. The Atlantic. 2020.

49. Rodríguez JE, Campbell KM, Pololi LH. Addressing disparities in academic medicine: what of the minority tax? BMC Medical Education. 2015;15(1):6. https://doi.org/10.1186/s12909-015-0290-9

50. White Coat for Black Lives Racial Justice Report Card. 2019.

51. Kowalczyk L. In an about-face, hospital will disperse portraits of past white male luminaries, put the focus on diversity. Boston Globe. 2018.

52. Torres MB, Salles A, Cochran A. Recognizing and Reacting to Microaggressions in Medicine and Surgery. JAMA surgery. 2019;154(9):868-72. https://doi.org/10.1001/jamasurg.2019.1648

53. Burgess DJ, Warren J, Phelan S, Dovidio J, van Ryn M. Stereotype Threat and Health Disparities: What Medical Educators and Future Physicians Need to Know. Journal of general internal medicine. 2010;25(2):169-77. https://doi.org/10.1007/s11606-009-1221-4

54. Ramani S, Könings KD, Ginsburg S, van der Vleuten CPM. Twelve tips to promote a feedback culture with a growth mind-set: Swinging the feedback pendulum from recipes to relationships. Medical Teacher. 2019;41(6):625-31. https://doi.org/10.1080/0142159X.2018.1432850

55. Ackerman-Barger $\mathbf{K}$, Valderama-Wallace $\mathbf{C}$, Latimore $\mathbf{D}$, Drake $\mathbf{C}$. Stereotype Threat Susceptibility Among Minority Health Professions Students. Journal of Best Practices in Health Professions Diversity. 2016;9(2):1232-46. https://doi.org/10.2307/26554256

56. Lai CK, Marini M, Lehr SA, Cerruti C, Shin JE, Joy-Gaba JA, et al. Reducing implicit racial preferences: I. A comparative investigation of 17 interventions. Journal of experimental psychology. General. 2014;143(4):1765-85. https://doi.org/10.1037/a0036260

57. Ufomata EA, Nandiwada R, Spagnoletti C, Bonnema R. Becoming antiracist in medical education: Embracing discomfort, acknowledging culpability, moving toward change. SGIM Forum. 2020.

58. Tsai J, Ucik L, Baldwin N, Hasslinger C, George P. Race Matters? Examining and Rethinking Race Portrayal in Preclinical Medical Education. Academic medicine : journal of the Association of American Medical Colleges. 2016;91(7):916-20. https://doi.org/10.1097/acm. 0000000000001232

59. Krishnan A, Rabinowitz M, Ziminsky A, Scott SM, Chretien KC Addressing Race, Culture, and Structural Inequality in Medical Education: A Guide for Revising Teaching Cases. Academic medicine : journal of the Association of American Medical Colleges. 2019;94(4):550-5. https:// doi.org/10.1097/acm.0000000000002589

60. Lupton KLOS, P. S. How can medical educators foster equity and inclusion in their teaching: A faculty development workshop series. Academic medicine : journal of the Association of American Medical Colleges. 2020; In press.

61. Lai CJ, Jackson AV, Wheeler M, Dhaliwal G, Ziv TA, Kryzhanovskaya I, et al. A framework to promote equity in clinical clerkships. The clinical teacher. 2020;17(3):298-304. https://doi.org/10.1111/tct.13050

62. Blakeney AM. Antiracist Pedagogy: Definition, Theory, and Professional Development. Journal of curriculum and pedagogy. 2005;2(1):119-32. https://doi.org/10.1080/15505170.2005.10411532

63. Hardeman RR, Medina EM, Boyd RW. Stolen Breaths. New England Journal of Medicine. 2020;383(3):197-9. https://doi.org/10.1056/ NEJMp2021072

64. A Toolkit for Teaching About Racism in the Context of Persistent Health and Healthcare Disparities Society of Teachers of Family Medicine Annual Spring Conference. 2017.

Publisher's Note: Springer Nature remains neutral with regard to jurisdictional claims in published maps and institutional affiliations. 